\title{
Rotational Thromboelastometry Helps to Reduce Blood Product Consumption in Critically III Patients during Small Surgical Procedures at the Intensive Care Unit - a Retrospective Clinical Analysis and Literature Search
}

\author{
Tomas Vymazal Marta Astraverkhava Miroslav Durila \\ Department of Anesthesiology and Intensive Care Medicine, University Hospital Motol, 2nd School of Medicine, Charles University, \\ Prague, Czech Republic
}

\section{Keywords}

Rotational thromboelastometry · ROTEM .

Fresh frozen plasma and platelet consumption .

Small surgical interventions at ICU

\section{Summary}

Background: Patients at intensive care units (ICUs) are often transfused to correct increased coagulation parameters (prothrombin time and activated partial thromboplastine time) and/or low platelet count. Thromboelastometry using whole blood is considered to be superior to these tests. In clinical praxis, prolonged standard tests are seen but thromboelastometry values are normal. The objective was to compare the blood product consumptions before and after the introduction of thromboelastometry assays into the treatment protocol during small surgical procedures at our mixed ICU. Methods: We analyzed 1,879 patients treated at our ICU who underwent small interventions. We compared the fresh frozen plasma and platelet consumption before and after the introduction of rotational thromboelastometry into the routine use. The obtained data were compared to relevant research results from the PubMed database, the MeSH index in the Medline database, and Google Scholar using key words 'tromboelastometry', 'fresh frozen plasma' and 'platelets'. Results: Annual fresh frozen plasma and platelet consumptions were significantly decreased following thromboelastometry introduction. The number of patients and procedures did not differ significantly during the periods analyzed. Conclusion: Routine thromboelastometry assays can enable significant reduction of blood product consumption in critically ill patients undergoing small surgery without any bleeding complications.

(C) 2018 S. Karger GmbH, Freiburg

\section{Introduction}

Critically ill patients are frequently exposed to the urge of small surgical interventions such as central venous catheter (CVC) introduction, chest drainage, surgical or percutaneous tracheostomy, or percutaneous gastrostomy (PEG) during in-hospital stay. Evaluation of extrinsic coagulation pathway using prothrombin time (PT) with its derived measures of prothrombin ratio (PR), international normalized ratio (INR) and platelet count is routinely required and used for coagulation assessment before invasive procedures in intensive care unit (ICU) patients. Because they do not reflect global coagulation profile (as they do not consider effect of platelets and other blood substances), they are being replaced by rotational thromboelastometry (ROTEM). Values of PT and/or aPTT and/or platelet count used to be often increased in critically ill patients due to many reasons [1-3]. Therefore, these patients receive inappropriate transfusions of blood components such as fresh frozen plasma (FFP) [1] and platelet concentrates (PCs) [4] to correct PT, aPTT and platelet count values $[5,6]$. The aim of our analysis was to prove, that ROTEM (ROTEM ${ }^{\circledR}$ International GmbH, Munich, Germany) can reduce blood product consumption in critically ill patients undergoing small surgery with no impact to bleeding complications following small surgical interventions.

\section{Material and Methods}

Our clinical analysis was performed retrospectively in two periods - without ROTEM measurements (April 2009 to April 2013) and following the ROTEM implementation (April 2013 to April 2015). The study comprised a sample of 1,879 patients. 523 of them were general medicine patients, 676 were post-surgery patients (abdominal and thoracic), and 680 were trauma patients. None of the patients had sepsis or septic shock. All patients were treated within 5-26 days. We focused on bleeding complications requiring surgical revisions, ultra-

\section{KARGER}

(c) 2018 S. Karger GmbH, Freiburg

Fax +497614520714 
Table 1. Characteristics of ICU patients

\begin{tabular}{lllllll}
\hline Time period & $2009-2010$ & $2010-2011$ & $2011-2012$ & $2012-2013$ & $2013-2014$ & $2014-2015$ \\
\hline Number of patients & 289 & 315 & 338 & 327 & 275 & 335 \\
Mean age, years & 67 & 69 & 66 & 71 & 69 & 68 \\
Male/female, \% & $68 / 32$ & $66 / 34$ & $62 / 38$ & $72 / 28$ & $68 / 32$ & $67 / 33$ \\
\hline
\end{tabular}

sound bleeding evidence, and/or chest tube insertion. Without ROTEM, FFPs and PCs were transfused prior to any small surgical procedures in order to correct abnormal standard coagulation test results, such as PT/INR and platelet count. According to hospital guidelines, the standard dose of FFP for the correction of increased PT/INR > 1.5 was $15 \mathrm{ml} / \mathrm{kg}$, resulting in approximately 4 IUs of FFP per patient on average. If the platelet count was lower than $50 \times$ $10^{9} / 1,2$ IUs of PCs were administered.

After implementation of ROTEM thrombelastometry, transfusion therapy was given according to the results of ROTEM measurement. If the PT/INR was $>1.5$, the platelet count was $>50 \times 10^{9} / 1$ and the aPTT was $<1.5$, the ROTEM EXTEM measurement was also performed. If the minimal values defining the normal range for clotting time (CT) in EXTEM (40-80 s) were reached, the surgical procedure was performed without any prophylactic FFP administration. If EXTEM testing showed pathological clotting times ( $>80 \mathrm{~s}), 4$ IUs of FFP were transfused prior to the procedure. In patients with platelet count $<50 \times$ $10^{9} / 1$ FIBTEM measurement was performed in addition to EXTEM due to the platelet function assessment. Then, the surgical procedure was performed without any prophylactic platelet administration if EXTEM and FIBTEM clot firmness (MCF) were within the normal ranges. In cases of low platelet count or dysfunction suggestion (lower MCF EXTEM and regular MCF FIBTEM), 2 IUs of PCs were administered prior to surgery.

Our analysis comprised all ICU patients who had an INR $>1.5$ or platelet count $<50 \times 10^{9} / 1$ prior to any small surgical procedure. None of the patients had any known pre-existing bleeding disorder and none was taking any antiplatelet and/or anticoagulant therapy. All patients received a prophylactic dose of the low-molecular weight heparin enoxarinum with the antiXa plasma level within $0.2-0.4 \mathrm{kIU} / 1$.

We compared our findings with relevant research results published in the PubMed database, the MeSH index in the Medline database, and Google Scholar. This was accomplished using the search terms: 'thromboelastometry', 'ROTEM', 'fresh frozen plasma', 'platelets', and 'reduction of consumption.' The NTT (number-to-treat) was set to 1,500. The GraphPad Prism statistics program (version 6.0, San Diego, CA, USA) was used to perform statistical analyses using descriptive statistics to determine median values.

\section{Results}

Mean annual FFP consumption based on standard coagulation tests was 6,235 units. ROTEM performance reduced it to 4,028 units (35\% reduction) in the first year and to 2,990 units (52\% reduction) during the next year. PC consumption based on ROTEM measurements decreased from 501 units to 487 units (3\% reduction) in the first and to 401 units (20\% reduction) in the next year. We found no bleeding complications requiring surgical revisions, ultrasound bleeding evidence, and/or chest tube insertion through the analyzed periods. The results are summarized in table 2.

\section{Discussion}

Routine coagulation tests measure blood plasma separated from blood cells. Thus, they do not reflect hemocoagulation as the whole. In contrast, the ROTEM measures the whole blood and
Table 2. Consumption of FFP and PCs before and after ROTEM institution

\begin{tabular}{lll}
\hline & $\begin{array}{l}\text { FFP consumption, } \\
\text { units }\end{array}$ & $\begin{array}{l}\text { Platelets consumption, } \\
\text { units }\end{array}$ \\
\hline $\begin{array}{ll}\text { Without ROTEM } \\
\text { 2009-2010 }\end{array}$ & \\
2010-2011 & 6,097 & 492 \\
2011-2012 & 7,809 & 531 \\
2012-2013 & 6,138 & 467 \\
Median 2009-2013 & 6,331 & 515 \\
With ROTEM & 6,235 & 503 \\
2013-2014 & & \\
2014-2015 & 4,028 & 487 \\
Median 2013-2015 & 2,990 & 401 \\
\hline
\end{tabular}

provides the unique and comprehensive way to assess coagulation, which reflects the physiologic conditions in vivo $[7,8]$. By using whole blood, it takes into consideration also platelet function which plays a critical role in coagulation [3]. That is why low level of coagulation factors (prolonged PT/aPTT) in cooperation with platelets is sufficient for good blood clotting. Vice versa, when there is low number of platelets, the high functional fibrinogen level (present in septic ICU patients) is sufficient for adequate clot formation [7]. Müller et al. [5] describe that patients with sepsis did not bleed during invasive procedures, despite increased values of INR. This phenomenon may be explained by the fact that whole blood has a different coagulation profile than pure plasma (when measuring INR/PR) and that blood cells such as platelets play critical role in coagulation as described in the cell-based model of coagulation. The findings of the current study are also similar to those of a randomized controlled trial published by De Pietri et al. [9] who showed that invasive procedures can be done without severe bleeding in patients with cirrhosis and INR $>1.8$. A possible limitation of ROTEM is that it is highly dependent on fibrinogen level. In most ICU patients the fibrinogen levels are increased [2]. Therefore, ROTEM is often normal despite of low platelet count and decreased INR.

It has been clearly proved that allogeneic FFP and PC transfusions carry high risks of bacterial contamination, allergic reactions, febrile reactions, venous thromboembolism, multiple organ failure, transfusion-related acute lung injury, transfusion-related circulatory overload [10], and poor wound healing [11] The strict avoidance of inappropriate FFP and PC transfusions might improve both the outcomes and prognoses of these patients as well as reduce transfusion-associated hospital costs [11]. Otherwise, the risk-benefit ratio of a FFP and PC transfusion strategy on the basis of abnormal laboratory test results in the absence of active bleeding is poorly understood $[6,12]$. Moreover, prolonged PT/aPTT and 
low platelet count not only do indicate hypocoagulation but also might accompany hypercoagulation [7]. Therefore, in case of normal ROTEM, we did not see any bleeding complication during small surgical procedures or afterwards despite prolonged PT/ aPTT or low platelets count [4]. The authors also demonstrated no bleeding complications in septic patients [13].

At our mixed ICU, ROTEM measurements decreased consumption of FFP by about $50 \%$ when compared to the time period when hemocoagulation was evaluated only by standard tests for PT/aPTT and platelet count. According to the literature, these data are in accordance with those of other authors who found ROTEM to be effective in reducing FFP and PC consumption in a cardiac intensive care unit [14], during liver transplantation [15], and also in management of traumatic bleeding [16] and massive hemorrhage management [17]. This approach to coagulation assessment protects patients from unnecessary FFP and PC administration which are generally connected with increased morbidity and mortality. A systematic review including 80 published randomized controlled clinical trials [18] has also shown no evidence of benefit in the prophylactic use of FFP and PC in non-bleeding ICU patients prior to surgery. Daily use of ROTEM is also costeffective [19].

\section{Conclusion}

ROTEM, as the global coagulation test, has changed our view on hemocoagulation and has helped us to provide the goal-directed and individually tailored therapy. When EXTEM results were within normal range, invasive procedures were performed without bleeding complications and without administration of FFP or other clotting factors, despite increased values of INR/PR. In spite of the general low risk of bleeding complications in small surgical procedures at ICUs, patients must be protected from any useless and potentially harmful transfusions of fresh frozen plasma and/or platelets. In addition, ROTEM contributes to spare financial resources and makes the treatment cost-effective.

\section{Retrospective Single-Center Analysis Limitations}

The main limitations of a retrospective single-center analysis are that significant biases may affect the selection of controls, may need very large sample sizes for rare outcomes, and can be very difficult to make accurate comparisons between the exposed and the non-exposed groups. Results from single-center studies are often 'site-specific' and hard to be widely accepted. In order to compensate these disadvantages, we analyzed a large cohort of 1,879 patients and compared our results with literature data .

\section{Authorship}

Marta Astraverkhava performed the research, analyzed the data, and participated in writing the paper.

Miroslav Durila designed and performed the research study.

Tomas Vymazal consulted the study, performed the literature search, and wrote the final version of the paper.

\section{Disclosure Statement}

None declared.

\section{References}

1 Walsh TS, Stanworth SJ, Prescott RJ, Lee RJ, Watson $\mathrm{DM}$ et al: Prevalence, management, and outcomes of critically ill patients with prothrombin time prolongation in United Kingdom intensive care units. Crit Care Med 2010;38:1939-1946.

2 Levi M, Opal,SM: Coagulation abnormalities in critically ill patients. Crit Care 2006;10:222.

3 Heemskerk JW, Mattheij NJ, Cosemans JM: Plateletbased coagulation: different populations, different functions. J Thromb Haemost 2013;11:2-16.

4 Gorlinger K, Saner FH: Prophylactic plasma and platelet transfusion in the critically ill patient: just useless and expensive or even harmful? BMC Anesthesiol 2015;15:86.

5 Müller MC, Arbous MS, Spoelstra-de Man AM, Vink $\mathrm{R}$, Karakus A et al: Transfusion of fresh-frozen plasma in critically ill patients with a coagulopathy before invasive procedures: a randomized clinical trial (CME). Transfusion 2015;55:26-35; quiz 25.

6 Holland L, Sarode R: Should plasma be transfused prophylactically before invasive procedures? Curr Opin Hematol 2006;13:447-451.

7 Saner FH: Rotational thrombelastometry: a step forward to safer patient care? Crit Care 2014;18:706.

8 Allen T: Thromboelastometry: its methodology, application, and benefits. MLO Med Lab Obs 2014;46:26-29.
9 De Pietri L, Bianchini M, Montalti R, et al: Thrombelastography guided blood product use before invasive procedures in cirrhosis with severe coagulopathy: a randomized, controlled trial. Hepatology 2016;63:566-573.

10 Kim KN, Kim DW, Jeong MA: The usefulness of a classification and regression tree algorithm for detecting perioperative transfusion-related pulmonary complications. Transfusion 2015:55:2582-2589.

11 Vymazal T, Horáček M, Duřpekt R, Hladíková M, Cvachovec K: Is allogeneic blood transfusion a risk factor for sternal dehiscence in cardiac surgery - a prospective observational study. Int Heart J 2009;50:601-608.

12 Gajic O, Dzik WH, Toy P: Fresh frozen plasma and platelet transfusion for nonbleeding patients in the intensive care unit: benefit or harm? Crit Care Med 2006; 34(5 suppl):S170-173.

13 Lukas P, Durila M, Jonas J, Vymazal T: Evaluation of thromboelastometry in sepsis in correlation with bleeding during invasive procedures. Clin Appl Thromb Hemost 2017; DOI: 10.1177/1076029617731624.

14 Anderson L, Quasim I, Soutar R, Steven M, Macfie A, et al: An audit of red cell and blood product use after the institution of thromboelastometry in a cardiac intensive care unit. Transfus Med 2006;16:31-39.
15 Görlinger K, Fries D, Dirkmann D, Weber CF, Hanke $\mathrm{AA}$, et al: Reduction of fresh frozen plasma requirements by perioperative point-of-care coagulation management with early calculated goal-directed therapy. Transfus Med Hemother 2012;39:104-113.

16 Schöchl H, Nienaber U, Maegele M, Hochleitner G, Primavesi F, et al: Transfusion in trauma: thromboelastometry-guided coagulation factor concentratebased therapy versus standard fresh frozen plasmabased therapy. Crit Care 2011:15:R83.

17 Vymazal T: Massive hemorrhage management - a best evidence topic report. Ther Clin Risk Manag 2015;11: 1107-1111.

18 Yang L, Stanworth S, Hopewell S, Doree C, Murphy M: Is fresh-frozen plasma clinically effective? An update of a systematic review of randomized controlled trials. Transfusion 2012;52:1673-1686.

19 Whiting P, Al M, Westwood M, Ramos IC, Ryder S, Armstrong $\mathrm{N}$,et al: Viscoelastic point-of-care testing to assist with the diagnosis, management and monitoring of haemostasis: a systematic review and cost-effectiveness analysis. Health Technol Assess 2015;19:1-228. 